\title{
On Improving the Performance of IEEE 802.11 with Multi-hop Concepts
}

\author{
Hao Zhu and Guohong Cao \\ Department of Computer Science \& Engineering \\ The Pennsylvania State University, University Park, PA 16802 \\ E-mail: \{hazhu,gcao\}@cse.psu.edu
}

\begin{abstract}
It is well known that IEEE 802.11 provides a physical layer multi-rate capability. In this paper, we propose a novel MAC layer relay-enabled point coordination function (PCF) protocol, called $r$ PCF, to exploit this capability. With multiple data rates in response to different channel conditions, if the direct link has low data rate, data packets may be delivered faster through a relay node. To enable MAC layer relay, the access point needs to collect information about the channel conditions, and notify mobile nodes which data rate to use and whether to use a relay node. We design protocols to achieve this goal and refine these protocols to minimize the control overhead. Simulation results show that $r$ PCF can significantly improve the system performance in terms of system throughput and transmission delay with a very small control overhead.
\end{abstract}

\section{INTRODUCTION}

With the dramatic development of wireless industries, wireless networking applications continue to proliferate at an incredible pace. With the advantage of low cost and high data rate, IEEE 802.11 wireless LANs (WLANs) are becoming increasingly popular, and constitute a large segment in the market of wireless products. Two different MAC mechanisms are supported by the IEEE 802.11 standard [5]: one is called distributed coordination function (DCF), which is based on carrier-sense multiple access with collision avoidance; the other is called point coordination function (PCF), which is based on polling. Due to the nature of contention-free, PCF outperforms DCF in terms of higher throughput and better QoS provisions [8]. Some efficient and robust polling schemes [1], [13] have been proposed to enhance the performance of PCF. In addition, it has been shown that PCF can achieve efficient power management [10]. Consequently, the polling-based PCF will be an attractive option for future WLANs.

IEEE 802.11 has physical-layer multi-rate capability [2], which means data can be transmitted at a number of data rates according to the channel condition. In practice, IEEE 802.11 b supports data rates of $1,2,5.5$, and $11 \mathrm{Mbps}$, and IEEE 802.11a supports data rates of $6,9,12,18, \ldots, 54$ Mbps. With the physical layer multi-rate capability, MAC layer mechanisms are required to exploit this capability. Several schemes [7], [4], [12] have been proposed to exploit the multirate capability under DCF. The basic idea of the schemes is to let the sender exploit the data rate according to the history of successful transmissions; or to let the receiver sense

This work was supported in part by the National Science Foundation (CAREER CCR-0092770 and ITR-0219711). the channel condition before the transmission, and notify the sender via the clear-to-send (CTS) packet. However, these schemes only exploit the data rate of the direct link between the sender and the receiver. In many cases, data may be delivered much faster through multiple links that have high data rates than through the direct link with low data rate.

In this paper, we propose a novel relay-enabled PCF $(r P C F)$ to exploit the multi-rate capability of IEEE 802.11 WLANs. Based on the channel condition, $r$ PCF may use multi-hop (mainly two-hop in this paper) data transmission through MAC layer relay to improve the system performance. When the direct link between the sender and the receiver has low data rate, whereas there exists a relay node such that both the links from the sender to the relay node and from the relay node to the receiver can support high data rate, the performance can be improved by letting the relay node forwarding the data packet from the sender to the receiver. To enable MAC layer relay, the access point needs to collect information about the channel conditions, and notify mobile nodes which data rate to use and whether to use a relay node. We design protocols to achieve this goal and refine these protocols to minimize the control overhead. Extensive simulations are provided and used to evaluate the proposed solution. Simulation results show that $r$ PCF can significantly improve the system performance in terms of system throughput and transmission delay, with only a negligible control overhead.

The remaining of this paper is organized as follows. Section II describes the background and motivation of the work. The details of $r \mathrm{PCF}$ are presented in Section III. Section IV evaluates the performance of $r$ PCF. Section $\mathrm{V}$ concludes the paper.

\section{BACKGROUND AND MOTIVATION}

\section{A. The System Model}

The system discussed in the paper is a typical basic service set (BSS) where all mobile stations are in the coverage area of the access point (AP). Each node, which refers to either a station or the AP, is equipped with a WLAN card such as [6] that enables the node to communicate with each other based on 802.11b. Although current 802.11 WLANs do not allow the direct communication between a pair of stations when the AP exists, following the standard [5] and without loss of the correctness, we still assume that the direct peerto-peer communication is enabled as long as two nodes are 
within the transmission range of each other. The AP mainly uses PCF to control the media access within the BSS and grants access to each station by polling. The physical layer uses direct-sequence spread spectrum (DSSS). Similar to [12], we model the data rates of $2 \mathrm{Mbps}, 5.5 \mathrm{Mbps}$ and $11 \mathrm{Mbps}$ for sending data packets in IEEE $802.11 \mathrm{~b}$. As specified by the standard [5], the data rate for sending physical-layer headers is set to be 1 Mbps. The control packets (i.e. poll, ACK, beacon, ...) and the broadcast packets are required to be transmitted at the rate of $2 \mathrm{Mbps}$, which uses QPSK modulation with very low bit error rate [4]. Although most WLANs allow roaming or hand-off, for simplicity, we do not consider this in the paper.

\section{B. The Point Coordination Function (PCF)}

PCF provides contention-free data transmission. Under PCF, the AP acts as the role of the point coordinator, maintains a polling list for eligible stations, and controls the medium access in a poll-and-response manner. Each station entering the coverage area of the WLAN needs to perform an association procedure [5] so that it can be added to the polling list by the AP. The period during which PCF operates is called the contention-free period (CFP). Before the CFP begins, the AP operates under DCF. Once the AP has announced the start of a CFP with a beacon frame, it may start transmitting data , or sending contention-free poll frames to the stations. During a CFP, a station can only transmit the impending data after being polled by the AP. If the polled station has data to send, it transmits one data packet. Otherwise, it will response the AP with a NULL packet. When the AP sends a packet to a station, it expects to hear a packet within a short inter-frame space (SIFS) [10]. If no packet is heard within a SIFS, the AP will reclaim the medium and send its next poll packet after a PIFS period [5]. The AP ends the CFP by sending a contention-free end packet.

\section{The Motivation}

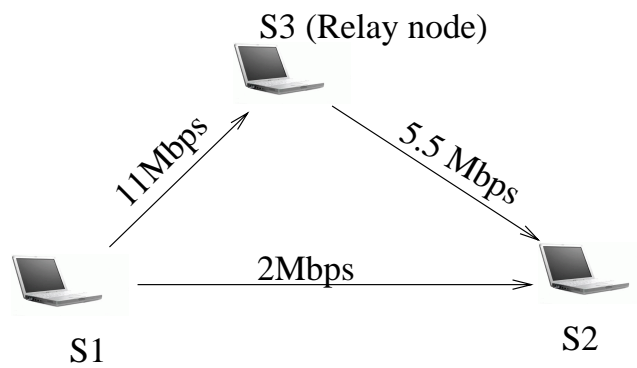

Fig. 1. The advantage of using the relay node

Since the channel condition of wireless networks is timevarying and location dependent [11], the multi-rate capability can be further exploited by enabling the MAC layer multi-hop transmission. For example, as shown in Figure 1, suppose $S_{1}$ needs to send data to $S_{2}$, and the channel of $S_{1} \rightarrow S_{2}$ only supports a data rate of $2 \mathrm{Mbps}$. At the same time, the channel conditions of $S_{1} \rightarrow S_{3}$ and $S_{3} \rightarrow S_{2}$ are much better, and they can support data rates of $11 \mathrm{Mbps}$ and $5.5 \mathrm{Mbps}$ respectively. If the data can be transmitted along $S_{1} \rightarrow S_{3} \rightarrow S_{2}$ at the MAC layer, suppose the packet length is $L$, the transmission delay is approximately equal to $\left(\frac{1}{5.5}+\frac{1}{11}\right) L m s$. Thus, the actual data rate is approximately equal to $\frac{5.5 \times 11}{5.5+11}=3.7 \mathrm{Mbps}$, which is much larger than 2 Mbps. To achieve this advantage, the relay node needs to relay the packet with the highest priority to avoid the queuing delay. Thus, the relaying operation should be performed at the MAC layer; that is, the relaying packet should not be treated the same as the data packets from upper layers. In this paper, we focus on two-hop MAC layer relay, which is sufficient in most of the cases.

To achieve the MAC layer relay mechanism, the key issue is to estimate the channel condition as precisely as possible before selecting the proper data rate and the way of the transmission. Since mobile stations have computation and power constraints and all stations are in the coverage area of the AP, the data rate selection could be done by the AP instead of the mobile stations. In particular, each station is required to sense the channel conditions of its links, and then the AP can collect the channel conditions of all stations, select the proper data rate and decide whether to use relay for the station being polled based on the collected information, and piggyback the rate selection decision with the polling packet.

\section{THE $r$ PCF PROTOCOL}

\section{A. Collecting the Channel Condition}

As mentioned in Section II-C, the AP is designed to perform the rate adaptation for the mobile stations. To achieve this goal, each station measures the channel condition, and the AP collects the channel condition measurements from each station. In particular, each station has a receive table, denoted by recv_table, to store the channel condition measurements of each link. Each entry of the recv_table is a tuple: < sender_id, rate $>$ and the content of each entry is determined as follows. When a station, say $S_{i}$, overhears a packet (by listening promiscuously), it measures the SNR when receiving the packet, and selects the proper date rate based on the measured SNR. It also takes the MAC layer header of the packet and uses the source address as the sender_id, and updates the corresponding rate field if the selected rate is different from the old one. If the sender_id does not exist in the recv_table, a new entry is added. If there is any change in recv_table, the station should notify the AP by sending a rate notification $(\mathrm{RN})$, which has a structure similar to that of the recv_table. In practice, a station only piggybacks the notification when it sends out an ACK or a data packet.

When $S_{i}$ receives a data packet or is polled but has no data to send, it checks if any entry in the recv_table has been changed since the last time of sending the RN. If there is any change, $S_{i}$ sends a RN piggybacked with the ACK or the NULL packet to the AP, and the AP extracts the RN from the packet. When $S_{i}$ is polled and has data packet to send, the situation becomes more complex. It is possible that the data packets are transmitted at a high rate (e.g., 11 Mbps), but the channel from $S_{i}$ to the AP can only support a low rate (e.g., 2 Mbps). Hence, the AP may not be able 
to extract the piggybacked $R N_{i}$ correctly. Without the up-todate channel condition from $S_{i}$, the AP may under-estimate or over-estimate the correspondent channel capacity, and the system performance will be degraded.

To deal with this problem, we use the following solution: When a station, say $S_{i}$, sends a data packet, to another station $S_{j}$, it piggybacks its $R N_{i}$ (if exists). When $S_{j}$ receives the packet, it checks the transmission rate as follows.

- If the transmission rate is equal to the lowest data rate, e.g., 2 Mbps in this paper, it replies an ACK piggybacked with $R N_{j}$ if there is any change in its recv_table.

- If the transmission rate is greater than the lowest rate, and $R N_{i}$ has been piggybacked with the data packet, $S_{j}$ sends $S_{i}$ an ACK which piggybacks both $R N_{i}$ and $R N_{j}$ (if exists).

As a result, if $R N_{i}$ is transmitted at a high data rate, it will be tunneled to the AP through $S_{j}$. Since the ACK is transmitted at $2 \mathrm{Mbps}$, the AP can successfully overhear the packet.

However, the solution alone is not enough. Since the AP may use out-of-date channel condition to estimate the data rate of $S_{i} \rightarrow S_{j}, S_{j}$ may not be able to correctly receive the $R N_{i}$. As a result, $S_{j}$ cannot receive the piggybacked $R N_{i}$, and tunnel it to the AP. To address this problem, we propose a new acknowledgment mechanism as follows:

- For $S_{i}$, if $S_{i}$ sends the data packet to $S_{j}$ and cannot hear the ACK within a SIFS, which means the transmission failed (otherwise, $S_{j}$ would reply the ACK), $S_{i}$ generates a NACK if it has $R N_{i}$ to send, and sends out the NACK piggybacked with $R N_{i}$ after a SIFS.

- For the AP, if it does not hear an ACK within a SIFS, it will send another poll if it still does not hear any packet within a PIFS.

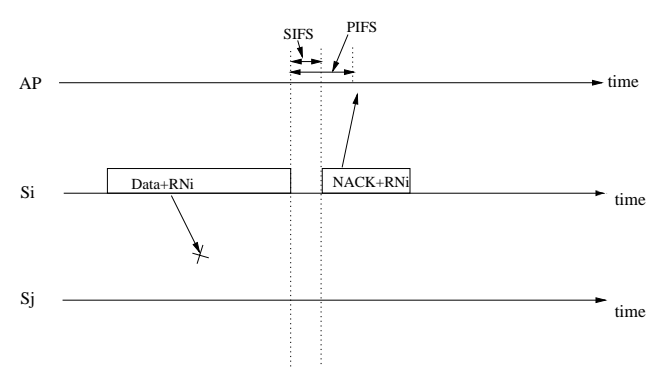

Fig. 2. An illustration of the sender-initiated NACK mechanism

This process has been illustrated in Figure 2. With the senderinitiated NACK, $R N_{i}$ can be delivered to the AP in time if all transmissions are successful at the lowest rate. After $S_{i}$ and the AP knows the failure, $S_{i}$ will adjust the data rate of $S_{i} \rightarrow S_{j}$ to the next lower rate, and the AP could poll $S_{i}$ again for re-transmission.

\section{B. The Rate Adaptation and Relay Mechanism}

In this section, we describe how the AP finds the proper data rate and how to relay packets at the MAC layer. Before the AP polls a station $S_{i}$, with the collected channel conditions from other stations, it estimates the channel condition and finds the proper rate for all possible receivers of $S_{i}$ because the AP does not know to which node $S_{i}$ will send. Suppose the AP stores the collected information into the rate table. in which an entry, denoted by $\hat{r}_{i j}$, is the estimated rate from node $\mathrm{i}$ to node $\mathrm{j}$ (a node refers to a station or the AP.) The AP can search the rate table and find out the set of potential receivers of $S_{i}$, denoted by $R_{i}$, according to:

$$
\mathcal{R}_{i}=\left\{\forall j \in B S S \mid \hat{r}_{i j} \neq N U L L\right\}
$$

where $B S S$ represents all nodes in the basic service set. However, $\hat{r}_{i j} \neq N U L L$ only implies that node $\mathrm{j}$ can overhear a packet from node $i$, which does not necessarily mean that node $\mathrm{j}$ will be the receiver of node $\mathrm{i}$. Also, finding and sending all possible data rates of the nodes in $\mathcal{R}_{i}$ may incur unnecessary computation and transmission overheads. In order to get a more accurate receiver set of each station, the AP relies on a learning process as follows: The AP maintains a flow table, denoted by $F T$, which contains the address pair of the sender and the receiver of each active flow in the system. In particular, when the AP overhears a data packet, and the address of the sender and receiver has not been registered in the FT, the AP adds the new address pair into the FT. With the FT, $\mathcal{R} i$ can be represented by:

$$
\mathcal{R}_{i}=\left\{\forall j \in B S S \mid \hat{r}_{i j} \neq N U L L \wedge<i, j>\in F T\right\}
$$

With $\mathcal{R}_{i}$, before the AP polls $S_{i}$, it looks up the rate table and finds the proper transmission rates for $S_{i}$. The transmission can be one-hop or two-hop. The data rate set of $S_{i}$, denoted by $D R_{i}$, is computed according to:

$$
\begin{aligned}
D R_{i}=\left\{\max \left(\hat{r}_{i j}, \max \left(\frac{\hat{r}_{i k} \times \hat{r}_{k j}}{\hat{r}_{i k}+\hat{r}_{k j}}\right)\right) \mid \forall k \in B S S \wedge j \in \mathcal{R}_{i}\right. \\
\left.\wedge \hat{r}_{i k}, \hat{r}_{k j} \neq N U L L\right\}
\end{aligned}
$$

Based on $\mathrm{Eq}(3)$, the AP selects the transmission with the highest data rate among all possible options. If the channel conditions do not change frequently, the content of two consecutive $D R_{i}$ s does not have too much difference. In order to reduce the transmission overhead, the AP only sends the difference between the current $D R_{i}$ and the previously computed $D R_{i}$. The data transmission notification can be piggybacked with the poll message. Each data transmission notification is a tuple: $<$ dest_id, 1 st hop rate, relay_id,2nd hop rate $>$. If the data rate is achieved by one-hop transmission, the relay ID and the second hop rate will not be needed.

Each station has a transmit table which caches the received data transmission notifications. When $S_{i}$ is polled, it updates its transmit table. If it has a data packet to send, it checks if there is an entry in the table whose destination ID matches the address of the receiver. If an entry is found, $S_{i}$ can get the correspondent transmission mode (one-hop or two-hop) and the data rate. Otherwise, it transmits the data packet at the lowest data rate.

According to the standard, each data packet only has two addresses: one for the sender and one for the receiver. In order to support two-hop MAC layer transmission, the address of the 
relay node needs to be added into the MAC layer header. With three different addresses, the relay mechanism is designed as follows. If a station receives a poll packet and finds out that the packet to send needs a two-hop transmission, for the first hop transmission, it sets the subtype of the packet to be the first hop transmission, and switches the addresses of the receiver and the relay node so that the packet is transmitted to the relay node first. When a node receives a packet with the subtype of the first hop transmission, it knows that it should relay the packet to the receiver. The relay node sets its address as the source address, sets the subtype of the packet to be the second hop transmission, and sends the packet to the receiver. When a node receives a packet with the subtype of the second hop transmission, it knows that it is the receiver. If the packet is correctly transmitted, the receiver replies an ACK directly to the original sender (Note that the address of the original sender is also stored in the header of the packet). If the packet is corrupted in either hop, the sender of that hop is able to know the error by overhearing if there is any packet transmitted within a SIFS, and reacts similarly to one-hop transmissions.

\section{Implementation Issues}

In this section, we describe how $r \mathrm{PCF}$ can be incorporated into IEEE 802.11. According to [5], each MAC frame has four address fields to indicate the BSS identifier (BSSID), source address (SA), destination address (DA), transmitting station address, and receiving station address. These addresses may appear in different order in different type of frames. In order to support $r \mathrm{PCF}$, some minor modifications to the standard 802.11 frames are required. Following the design rationale, the modifications are listed as follows:

1) A new data frame format is defined to support MAC layer relay. Specifically, each data frame uses all four address fields in the order of SA, DA, BSSID, and fourth address. The first and second hop relay can be differentiated by the subtype value in the frame control field. For data type, which is indicated by type value of 10 (binary), subtype value can be selected from the reserved ones from 1000 to 1111. For twohop transmissions, with the SA, DA and fourth address fields, the addresses of the sender, the relayer and the receiver can be stored in the packet frame.

2) In order to identify the piggybacked notifications, we add an 8 bits notification tag in the frame. The value of the tag indicates the number of rate notifications or data transmission notifications, which are piggybacked with the frame.

3) In order to collect and estimate the channel condition of its neighbors, each station needs to know the source address of each packet transmitted. Thus, the standard IEEE 802.11 ACK frame format has been changed to include the source address.

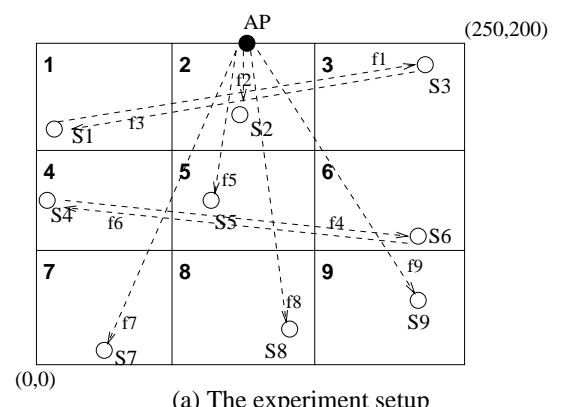

(a) The experiment setup

\begin{tabular}{|c|c|c|c|c|l|l|l|l|l|l|}
\hline $\mathrm{ID}$ & $\mathrm{AP}$ & $\mathrm{S} 1$ & $\mathrm{~S} 2$ & $\mathrm{~S} 3$ & $\mathrm{~S} 4$ & $\mathrm{~S} 5$ & $\mathrm{~S} 6$ & $\mathrm{~S} 7$ & $\mathrm{~S} 8$ & $\mathrm{~S} 9$ \\
\hline $\mathrm{x}$ & 125 & 13 & 112 & 223 & 9 & 96 & 224 & 35 & 96 & 237 \\
\hline $\mathrm{y}$ & 200 & 139 & 163 & 192 & 105 & 92 & 70 & 11 & 36 & 59 \\
\hline
\end{tabular}

(b) The coordinates of each node

Fig. 3. The experiment setup

\section{The Performance Evaluations}

\section{A. The Simulation Setup}

Our simulation is based on ns-2 extension [9], [3]. The experiment setup is shown in Figure 3(a). As shown in Figure $3(\mathrm{a})$, one AP and nine stations exist in a $250 \times 200$ flat area. The coordinates of the nodes are listed in Figure 3(b). We assume the stations are evenly distributed in the area. We divide the area into 9 subareas indexed from 1 to 9 , and the mobile station with the same index resides in the correspondent subarea. Each station has a flow, starting either from another station or from the AP. If the connection is from one station to another, the flow number is the same as the index of the sender (e.g., $f_{1}$ represents the connection from $S_{1} \rightarrow S_{3}$.) If the connection is between the AP to a station, the flow number is the same as the index of the receiver (e.g., $f_{2}$ represents the connection from $A P \rightarrow S_{2}$.) Similar to [12], the distance threshold for $11 \mathrm{Mbps}, 5.5 \mathrm{Mbps}$, and $2 \mathrm{Mbps}$ are $100 \mathrm{~m}, 200 \mathrm{~m}$, and $250 \mathrm{~m}$ respectively. The thresholds for different data rates are chosen based on the distance ranges [10]. For simplicity, all flows in the system are assumed to have the same type of traffic source with the same rate. There are two kinds of traffic configurations: 1) each sender always has data to send; 2) each sender has CBR traffic with the rate of $20 \mathrm{packet} / \mathrm{second}$. The packet length is set to be 1000 bytes and the simulation time is assumed to be 500 seconds.

We use the Friis free space propagation model and the 2ray ground propagation model as the large-scale propagation models to simulate the situation when the wireless channel condition is stable. The two model are combined by using the Friis model at short distance and the 2-way ground model at long distance. The crossover distance $d_{\text {cross }}$ is calculated by $d_{\text {cross }}=\frac{4 \pi h_{r} h_{t}}{\lambda}$, where $h_{t}, h_{r}$ are the transmit and receive antenna heights (in meters), and $\lambda$ is the carrier wavelength (in meters). When there is multi-path fading or relative movement between the sender and receiver, the channel condition between them may change frequently. The frequency of this change depends on the relative speed of the mobile node with 


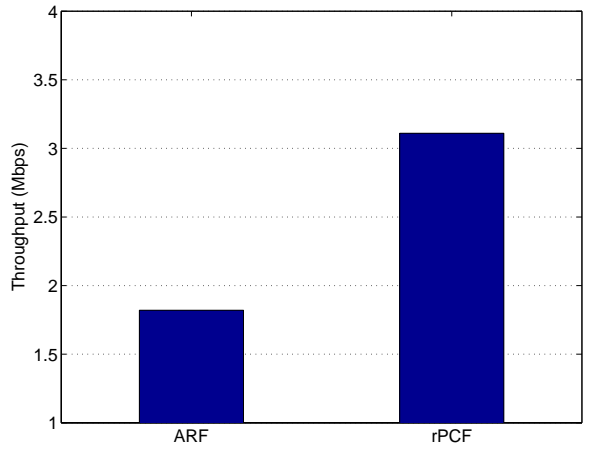

(a) System Throughput
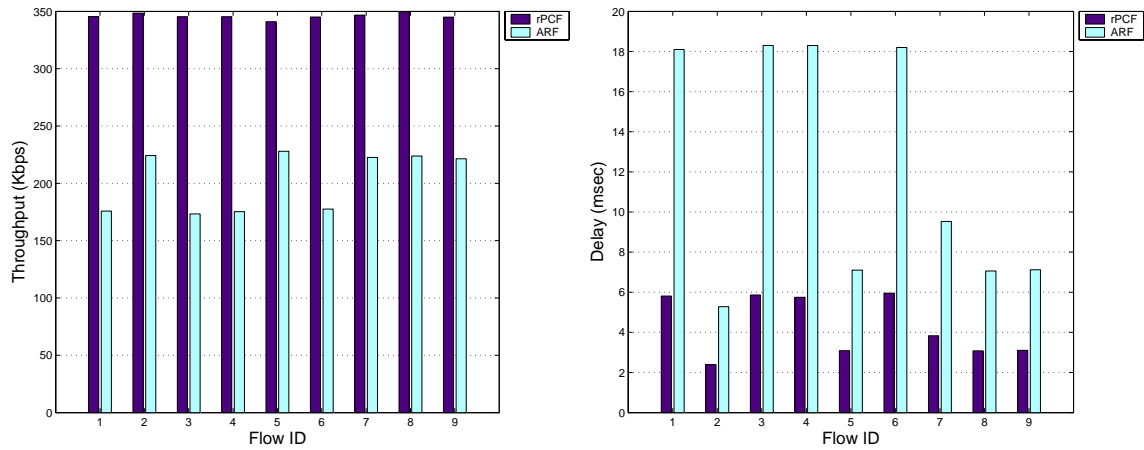

(b) Throughput of each flow

(c) Delay of each flow (rate=20pkt/s)

Fig. 4. Performance comparisons between $r \mathrm{PCF}$ and $\mathrm{ARF}$

respect to its surroundings. We use the Ricean fading model [11] to simulate the fading channel conditions. The Ricean distribution is given by:

$$
p(r)=\frac{r}{\sigma^{2}} e^{-\left(\frac{r}{2 \sigma^{2}}+K\right)} I_{0}(2 K r)
$$

where $K$ is the distribution parameter representing the lineof-sight component of the received signal, $\sigma^{2}$ is the variance of the background noise, $r$ is the received power, and $I_{0}($.$) is$ the modified Bessel function of the first kind and zero order [11].

We compare $r \mathrm{PCF}$ with PCF-based ARF [7], which is a sender-based rate adaptation scheme. The protocol works as follows. If ACKs for two consecutive data packets are lost, the sender reduces the transmission rate to the next lower data rate and starts a timer. If ten consecutive ACKs are received, the transmission rate is raised to the next higher data rate and the timer is cancelled. If the timer expires, the transmission rate is raised if an ACK is received for the next packet; otherwise, the rate is lowered again and the timer is restarted. Since the simulation results of [4] show that the throughput of ARF does not change much when the timeout is larger than $60 \mathrm{~ms}$, we set the timeout value to be $80 \mathrm{~ms}$ in our simulations.

\section{B. Simulation Results}

We evaluate the impacts of different factors on the system performance, which is measured by the system throughput and the average packet transmission delay. We also evaluate the system overhead of the proposed solution.

1) Performance under Stable Links: In this subsection, we assume the channel conditions are stable, so that the received power is calculated only by the Friis and 2-ray ground propagation models. Further, all stations are assumed to be static. In this way, we can maximize the performance of different schemes. The simulation results are shown in Figure 4. From Figure 4(a), we can see that $r$ PCF improves the system throughput by more than $70 \%$. The performance gain is mainly due to the MAC layer relay. For example, the transmission rate for $f_{1}$ is limited to $2 \mathrm{Mbps}$ under ARF due to the long distance between $S_{1}$ and $S_{3}$. Under $r \mathrm{PCF}$, the packets of $f_{1}$ can be relayed by the AP. Because the distances between $S_{1}$ and the AP, $S_{3}$ and the AP are both less than $100 \mathrm{~m}$, the data rates of $S_{1} \rightarrow A P$ and $A P \rightarrow S_{3}$ are both $11 \mathrm{Mbps}$, and hence the actual data rate of $f_{1}$ is approximately 5.5 Mbps. As shown in Figure 4(c), under ARF, the delays of $f_{1}, f_{3}$, $f_{4}$, and $f_{6}$ are much higher than the rest of the flows. The reasons are as follows. Let us take $f_{1}$ as the example, ARF raises the transmission rate of $f_{1}$ to $5.5 \mathrm{Mbps}$ after $S_{1}$ receives the ACKs for ten consecutive packets. Then, raising the data rate will cause $f_{1}$ 's next packet to fail since the link quality between $S_{1}$ and $S_{3}$ cannot support $5.5 \mathrm{Mbps}$. In addition, ARF reduces the transmission rate of $f_{1}$ back to 2 Mbps only when $S_{1}$ misses two ACKs for two consecutive data packets. This leads to extra delay of $f_{1}$ due to retransmissions. Furthermore, the extra delay also affects the delay of other flows (e.g., $f_{2}$ ) since the AP polls each station in a round robin way.

2) Impacts of the Line-of-sight parameter $K$ : When the channel condition is stable and the stations do not move, the AP and the mobile stations only send a very limited number of rate notifications (RNs) and data transmission notifications (TNs), and the overhead of $r \mathrm{PCF}$ is negligible. Since the wireless channel condition may change frequently, the overhead of $r$ PCF, which includes transmitting RNs, TNs, and NACKs, should be evaluated. To evaluate this overhead, we change the line-of-sight parameter $K$. A large $K$ means a better channel quality while a small $K$ indicates a poor channel quality. The sizes of RN, one-hop TN, two-hop TN and NACKs are conservatively assumed to be 56, 56, 112 and 304 (in bits) respectively. We collect the generated RNs, TNs and NACKs, and add them together to get the control overhead. Then, using these control overhead (in bits) divided by the simulation time, we get the control overhead in terms of data rate (bps). Since ARF does not have any control overhead, we introduce a new metric called cost gain percentage (CGP) to evaluate the control overhead of $r$ PCF fairly. The CGP (in percent) is the ratio of the control overhead (bps) to the throughput gain of $r \mathrm{PCF}$ (compared to ARF).

The simulation results are shown in Figure 5. From Figure 5(a), we can see that the system throughput of both $r \mathrm{PCF}$ and ARF increase as $K$ increases. As $K$ increases, the impact of fading becomes smaller, and the channel condition becomes more stable. As a result, the system throughput improves. 


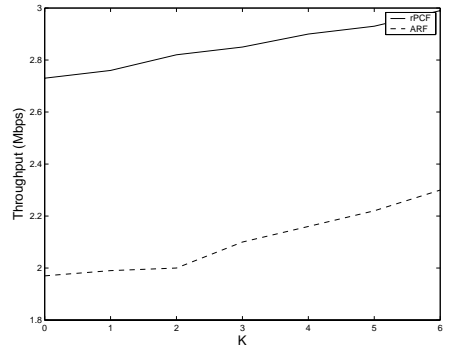

(a) Throughput of ARF and $r \mathrm{PCF}$

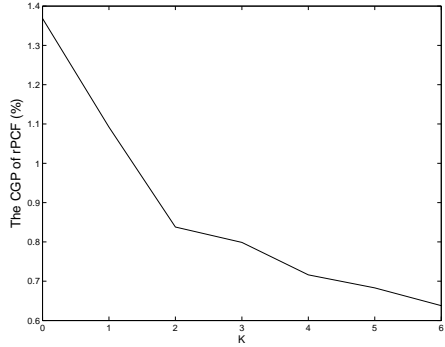

(b) The CGP of rPCF
Fig. 5. Impacts of the line-of-sight parameter $K$

From the figure, we can also see that the impact of $K$ on the throughput of ARF is higher than that on $r \mathrm{PCF}$. When the channel condition becomes unstable, the total number of mis-adaptations under ARF is much greater than that under $r$ PCF. However, under $r \mathrm{PCF}$, each station frequently reports the channel conditions to the AP, so that the AP can perform rate adaptation and notify correspondent stations in time. As a result, stations can adjust the transmission rates in time and reduce the chance of using wrong data rates. As shown in Figure 5(b), the control overhead of $r$ PCF drops quickly as $K$ increases. From the results shown in Figure 5(b), we can see that the control overhead is very small. For example, the CGP is only about $1.3 \%$ when $K=0$, and drops to $0.6 \%$ when $K=6$.

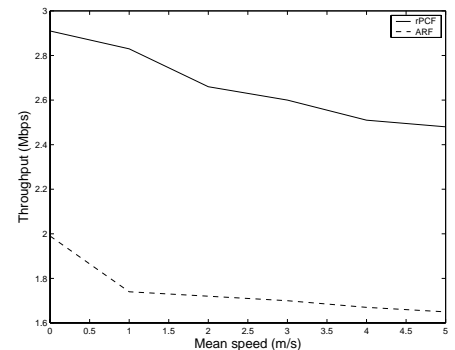

(a) Throughput of $\mathrm{ARF}$ and $r \mathrm{PCF}$

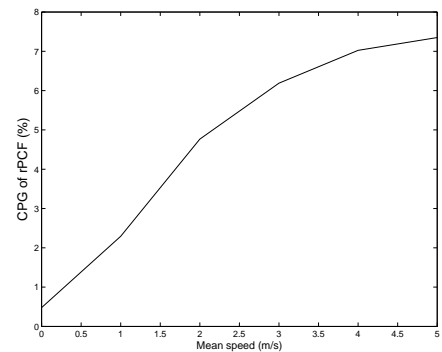

(b) The CGP of rPCF
Fig. 6. Impacts of mobility

3) Impacts of Mobility: Mobility affects the channel condition in two aspects: First, it changes the node's location which may affect the value of $K$ and the strength of the received signal power. Second, since node mobility causes a Doppler shift in the frequency of the received signal, it may reduce the channel coherence time period, which is the interval during which the channel condition keeps stable. In this subsection, we evaluate the impact of mobility on the performance of $r$ PCF. Each station is assumed to move randomly within the correspondent subarea with the same speed, and the mean speed changes from 0 to $5 \mathrm{~m} / \mathrm{s}$. Similar to [12], the value of $K$ is fixed to be 5.0. The simulation results are shown in Figure 6. As shown in Figure 6 (a), the system throughput of $r$ PCF and ARF drops as the mean moving speed increases. As the moving speed increases, the channel condition changes frequently. When the channel condition becomes unstable (e.g. when the mean speed is $5 \mathrm{~m} / \mathrm{s}$ ), it is difficult for ARF to adjust the transmission rate to a high rate, and hence most of the data are transmitted at a low rate of $2 \mathrm{Mbps}$. For $r \mathrm{PCF}$, the increased control overhead such as NACKs, RNs and TNs also reduces the system throughput as the moving speed increases. As shown in Figure 6 (b), the control overhead is still very small. For example, when the mean speed is $5 \mathrm{~m} / \mathrm{s}$, the CGP is only about $7.7 \%$.

\section{CONClusions And Future Work}

In this paper, we presented a novel MAC layer relay-enabled PCF protocol, called $r \mathrm{PCF}$, to exploit the physical layer multirate capability. Since IEEE 802.11 supports multiple data rates in response to different channel conditions, data packets may be delivered faster through a relay node than through the direct link if the direct link has low quality and low rate. Several protocols and their optimizations are proposed to help the AP collect the up-to-date channel information and notify mobile nodes what data rate to use and whether to use a relay node. Simulation results showed that $r$ PCF outperforms PCFbased ARF in terms of throughput and transmission delay with very small control overhead. In the future, we will extend the relay mechanism to DCF or the emerging Hybrid Coordination Function of IEEE 802.11e.

\section{REFERENCES}

[1] A. Ganz, A. Phonphoem and Z. Ganz, "Robust superpoll with chaining protocol for IEEE 802.11 wireless LANs in Support of Multimedia Applications," Wireless Networks, 2001.

[2] Jim Geier, "Wireless LANs, 2nd Edition," Sams Publishing, 2002.

[3] VINT group, "UCB/LBNL/VINT Network Simulator - ns (Version 2)," http://mash.cs.berkeley.edu/ns.

[4] G. Holland, N. Vaidya and P. Bahl, "A rate-adaptive MAC protocol for multi-hop wireless networks," ACM Mobicom 2001, July 2001.

[5] IEEE, "Wireless LAN Medium Access Control (MAC) and Physical Layer (PHY) Sp ec," IEEE 802.11 standard, 1999.

[6] Agere Systems Inc., "Wireless LAN Products and Technologies ," http://www.agere.com/client/wlan.html.

[7] A. Kamerman and L. Monteban, "WLAN-II: A high-performance wireless LAN for the unlicecsed band," Bell Labs Technical Journals, summer 1997.

[8] A. Kospel, J. P. Ebert and A. Wolisz, "A performance comparison of point and distributed coordination function of an IEEE 802.11 WLAN in the presence of real-time requirements," 7th International Workshop on Mobile Multimedia Communication (MoMuC2000), Oct. 2000.

[9] R. Punnoose, P. Nikitin and D. Stancil, "Efficient simulation of Ricean fading within a packet simulator," IEEE VTC 2000, pp. 764-767, 2000.

[10] D. Qiao, S. Choi, A. Soomro and K. G Shin, "Energy-Efficient PCF Operation of IEEE 802.11a Wireless LAN," IEEE INFOCOM'02, June 2002.

[11] Theodore S. Rappaport, "Wireless Communications: Principle and Practice," Prentice Hall, 1996.

[12] B. Sadeghi, V. Kanodia, A. Sabharwal and E. Knightly, "Opportunistic media access for multirate Ad Hoc networks," ACM Mobicom 2002, July 2001

[13] O. Sharon and Eitan Altman, "An efficient polling MAC for Wireless LANs," IEEE/ACM Transactions on Networking, Aug. 2001. 